\title{
Determination of the Babuska-Aziz Constant for the Linear Triangular Finite Element
}

\author{
Fumio KikUCHI and LiU Xuefeng \\ Graduate School of Mathematical Sciences, University of Tokyo, \\ 3-8-1 Komaba, Meguro, Tokyo 153-8914, Japan
}

Received March 30, 2005

Revised September 16, 2005

\begin{abstract}
We explicitly determine the Babuska-Aziz constant, which plays an essential role in the interpolation error estimation of the linear triangular finite element. The equation for determination is the transcendental equation $t+\tan t=0$, so that the solution can be numerically obtained with desired accuracy and verification. Such highly accurate approximate values for the constant can be widely used for a priori and a posteriori error estimations in adaptive computation and/or numerical verification.
\end{abstract}

Key words: linear triangular finite element, Babuska-Aziz constant, interpolation error estimation

\section{Introduction}

The finite element method (FEM) is now recognized as a powerful numerical method for wide classes of partial differential equations. Furthermore, it also has sound mathematical bases such as highly refined a priori and a posteriori error estimations. In the classical a priori error analysis of FEM, interpolation errors are essential to derive final error estimates $[4,5]$. In this process, there appear various positive constants besides the standard discretization parameter $h$ and norms (or seminorms), but it has been very difficult to evaluate such constants explicitly. For quantitative purposes, however, it is indispensable to evaluate or bound them as accurate as possible. Thus such evaluation has been attempted for adaptive finite element calculations based on a posteriori error estimation as well as for numerical verification by FEM. In this paper, we will determine the so-called Babuska-Aziz constant [2], which appears in the estimation of the interpolation errors of the linear $\left(P_{1}\right)$ triangular finite element.

More specifically, we derive a transcendental equation for the above constant. To this end, we use the reflection (or symmetry) method to solve analytically an eigenvalue problem for 2D Laplace operator. As we will see later, this constant $\left(C_{1}\right)$ gives an upper bound to the optimal constant $\left(C_{0}\right)$ appearing in the $H^{1}$-error estimation of $P_{1}$-interpolation functions for $H^{2}$-functions over the unit isosceles right triangle. The $P_{1}$-finite element is the most classical and fundamental one, but still in frequent use. Thus precise estimation of $C_{1}$ as well as $C_{0}$ is very important, and a number of researchers have given bounds for these two constants using various approximation methods including numerical verification, see e.g. [1, $7,8,9,10,11]$. The relation between $C_{0}$ and $C_{1}$ was pointed out by Babuska and 
Aziz in conjunction with the maximum angle condition [2], and later discussed in $[8,11]$.

The transcendental equation for the required eigenvalue $\lambda\left(=1 / C_{1}^{2}\right)$ is a very simple one given by $\sqrt{\lambda}+\tan \sqrt{\lambda}=0$. Thus the constant can be easily obtained with sufficient accuracy, and can be effectively used in the quantitative error estimation of finite element solutions by the $P_{1}$-element.

\section{Preliminaries}

Let $T$ be a unit right-angled isosceles triangle defined by $T=\left\{x=\left(x_{1}, x_{2}\right) \in\right.$ $\left.\mathbf{R}^{2} ; x_{1}>0, x_{2}>0, x_{1}+x_{2}<1\right\}$, the vertices of which are denoted by $Q_{1}(0,0)$, $Q_{2}(1,0)$ and $Q_{3}(0,1)$. Let us define the sets $V_{1}$ and $V_{2}$ by

$$
\begin{aligned}
& V_{1}=\left\{v \in H^{2}(T) ; v\left(Q_{1}\right)=v\left(Q_{2}\right)=v\left(Q_{3}\right)=0\right\}, \\
& V_{2}=\left\{v \in H^{1}(T) ; \int_{0}^{1} v\left(x_{1}, 0\right) d x_{1}=0\right\},
\end{aligned}
$$

where $H^{1}(T)$ and $H^{2}(T)$ are respectively the first- and second-order Sobolev spaces of real square integrable functions over $T$. Furthermore, the space $L_{2}(T)$ equipped with norm $\|\cdot\|_{T}$ will be also used. Notice here that $\partial v / \partial x_{1} \in V_{2}$ for $\forall v \in V_{1}$.

For $v \in H^{2}(T)$, the linear interpolation function $\Pi v$ on $T$ is the (at most) linear polynomial such that $(\Pi v)\left(Q_{i}\right)=v\left(Q_{i}\right)$ for $1 \leq i \leq 3$. Then $v-\Pi v \in V_{1}$ for $v \in H^{2}(T)$, so that a popular form of the interpolation error for $v$ is $([4,5])$

$$
|v-\Pi v|_{1, T} \leq C_{0}|v|_{2, T},
$$

where $|\cdot|_{1, T}$ and $|\cdot|_{2, T}$ are the usual seminorms of $H^{1}(T)$ and $H^{2}(T)$, respectively: $|v|_{1, T}^{2}=\sum_{i=1}^{2}\left\|\partial v / \partial x_{i}\right\|_{T}^{2},|v|_{2, T}^{2}=\sum_{i, j=1}^{2}\left\|\partial^{2} v / \partial x_{i} \partial x_{j}\right\|_{T}^{2}$. Moreover, $C_{0}$ is the (optimal) positive constant well-defined by the relation $[1,2,8]$

$$
C_{0}=\sup _{v \in V_{1} \backslash\{0\}} \frac{|v|_{1, T}}{|v|_{2, T}} .
$$

Estimation (3) is effectively used for error analysis for triangular elements of more general shape by introducing appropriate coordinate transformations $[2,4,5,6]$.

It is actually difficult to decide $C_{0}$ exactly. An upper bound for $C_{0}$ was first given by Natterer [10]. By numerical computations without verification, it is now known that $C_{0} \approx 0.489$, cf. $[1,7,11]$. Moreover, $C_{0}$ has an upper bound $C_{1}$ given by

$$
C_{1}=\sup _{v \in V_{2} \backslash\{0\}} \frac{\|v\|_{T}}{|v|_{1, T}} .
$$

This constant was introduced by Babuska and Aziz in [2] to prove the maximum angle condition for the $P_{1}$-element, so that we call it the Babuska-Aziz constant here. The relation between $C_{0}$ and $C_{1}$ was fully discussed in [11] and [8], and in 
certain cases $C_{1}$ is more essential than $C_{0}$ itself (see below). In particular, $C_{1}$ was verified numerically in $[8,9]$, and it is now known that $0.492 \leq C_{1} \leq 0.494$. Thus 0.493 or so is a nice approximation to $C_{0}$ for most of practical purposes: In fact, 0.5 is recommended in [11] for use as an upper bound for $C_{0}$.

Furthermore, $C_{1}$ plays its own role of enabling estimation of each partial derivative:

$$
\left\|\partial(v-\Pi v) / \partial x_{i}\right\|_{T} \leq C_{1}\left|\partial v / \partial x_{i}\right|_{1, T} \quad(i=1,2),
$$

which is in a sense sharper than (3), cf. [6].

It is easily seen that $C_{1}$ is determined as $C_{1}=\sqrt{1 / \lambda_{0}}$, where $\lambda_{0}>0$ is the minimum eigenvalue of the eigenvalue problem [1, 8]: Find $\lambda$ and $u \in V_{2} \backslash\{0\}$ that satisfy

$$
(\nabla u, \nabla v)_{T}=\lambda(u, v)_{T} \quad\left(\forall v \in V_{2}\right)
$$

Here, $(\cdot, \cdot)_{T}$ denotes the inner products of both $L_{2}(T)$ and $L_{2}(T)^{2}$, and $\nabla$ is the gradient operator. The present eigenvalue problem is also expressed as follows in terms of a partial differential equation, the linear constraint for $V_{2}$ and boundary conditions $[1,8]$ :

$$
-\Delta u=\lambda u \text { in } T, \int_{0}^{1} u\left(x_{1}, 0\right) d x_{1}=0, \quad \frac{\partial u}{\partial n}= \begin{cases}0 & \text { on edges } Q_{1} Q_{3} \text { and } Q_{2} Q_{3} \\ c & \text { on edge } Q_{1} Q_{2}\end{cases}
$$

where $\frac{\partial}{\partial n}$ denotes the outward normal derivative on edges, and $c$ an unknown constant. More specifically, $c$ must finally satisfy the relation $c+$ $\lambda \iint_{T} u\left(x_{1}, x_{2}\right) d x_{1} d x_{2}=0$. Thus, $c=0$ if $\iint_{T} u\left(x_{1}, x_{2}\right) d x_{1} d x_{2}=0$. Otherwise, $c$ can be equated to 1 at the expense of imposing the condition $\iint_{T} u\left(x_{1}, x_{2}\right) d x_{1} d x_{2}=$ $-1 / \lambda$ on the magnitude of $u[8]$.

\section{Determination of the Constant}

Our problem of determining $C_{1}$ now reduces to obtaining the minimum eigenvalue of (7) or (8). Since $T$ is a triangular domain, it has been not necessarily easy to solve the associated eigenvalue problem. However, we have the following main results.

THEOREM 1. The minimum eigenvalue $\lambda_{0}$ of (7) is equal to the minimum positive solution of the transcendental equation for $\lambda$ :

$$
\sqrt{\lambda}+\tan \sqrt{\lambda}=0
$$

The concrete value of $\lambda_{0}$ can be obtained numerically with verification. For example, $\sqrt{\lambda}_{0}$ lies in the interval $2.0287<\sqrt{\lambda}_{0}<2.0291$, and hence $C_{1}=1 / \sqrt{\lambda}_{0}$ is bounded as

$$
0.49282<C_{1}<0.49293
$$


(Numerical computation without verification gives $C_{1}=0.49291245 \cdots$.)

Proof. We will prove in several steps, each of which is based on rather wellknown arguments and techniques, and is sometimes described concisely.

$1^{\circ}$. Let $\Omega$ be a unit square domain: $\Omega=\left\{x=\left(x_{1}, x_{2}\right) \in \mathbf{R}^{2} ; 0<x_{1}, x_{2}<1\right\}$. Let $\{\lambda, u\} \in \mathbf{R} \times V_{2} \backslash\{0\}$ be an arbitrary eigenpair of (7), and define the (symmetric) extension $\tilde{u}$ of $u$ to $\Omega$ by reflection with respect to the line $x_{1}+x_{2}=1$ :

$$
\begin{aligned}
& \tilde{u}\left(x_{1}, x_{2}\right)=u\left(x_{1}, x_{2}\right) \text { if } x=\left(x_{1}, x_{2}\right) \in T, \\
& \tilde{u}\left(x_{1}, x_{2}\right)=u\left(1-x_{2}, 1-x_{1}\right) \quad \text { if } \quad x \in \Omega \backslash T .
\end{aligned}
$$

It is easy to see that $\tilde{u}$ belongs to $H^{1}(\Omega)$. Moreover, $\{\lambda, \tilde{u}\}$ becomes an eigenpair of the eigenvalue problem for $\Omega$ :

$$
\tilde{u} \in \tilde{V}_{2} \backslash\{0\} \text { and }(\nabla \tilde{u}, \nabla \tilde{v})_{\Omega}=\lambda(\tilde{u}, \tilde{v})_{\Omega} \quad\left(\forall \tilde{v} \in \tilde{V}_{2}\right),
$$

where $(\cdot, \cdot)_{\Omega}$ denotes the inner products of $L_{2}(\Omega)$ and $L_{2}(\Omega)^{2}$, and $\tilde{V}_{2}$ is defined by

$$
\tilde{V}_{2}=\left\{\tilde{v} \in H^{1}(\Omega) ; \int_{0}^{1} \tilde{v}\left(x_{1}, 0\right) d x_{1}=0, \int_{0}^{1} \tilde{v}\left(1, x_{2}\right) d x_{2}=0\right\} .
$$

Conversely, any eigenpair of (a) with $\tilde{u}$ restricted to $T$ satisfies (7), if $\tilde{u}$ is symmetric with respect to the line $x_{1}+x_{2}=1$. Notice here the orthogonal decomposition of $\tilde{V}_{2}$ in $H^{1}(\Omega)$ as well as in $L_{2}(\Omega)$ :

$$
\tilde{V}_{2}=\tilde{V}_{2}^{s} \oplus \tilde{V}_{2}^{a}, \quad\left\{\begin{array}{l}
\tilde{V}_{2}^{s}=\text { subspace of symmetric functions in } \tilde{V}_{2}, \\
\tilde{V}_{2}^{a}=\text { subspace of antisymmetric functions in } \tilde{V}_{2} .
\end{array}\right.
$$

Consequently, for the present purpose, it suffices to deal with (a) in $\tilde{V}_{2}^{s}$.

$2^{\circ}$. As is well known, a complete system of functions for $H^{1}(\Omega)$ is given by the totality of (orthogonal) eigenfunctions of (a):

$$
\phi_{m n}\left(x_{1}, x_{2}\right)=\cos m \pi x_{1} \cos n \pi x_{2} \quad(m, n=0,1,2,3, \ldots) .
$$

Since we are interested in symmetric eigenfunctions only, we should make a complete system of symmetric functions in $H^{1}(\Omega)$ from the above: for $m \geq n ; m, n=$ $0,1,2,3, \ldots$,

$$
\begin{aligned}
\psi_{m n}\left(x_{1}, x_{2}\right) & =\phi_{m n}\left(x_{1}, x_{2}\right)+\phi_{m n}\left(1-x_{2}, 1-x_{1}\right) \\
& =\phi_{m n}\left(x_{1}, x_{2}\right)+(-1)^{m+n} \phi_{n m}\left(x_{1}, x_{2}\right) .
\end{aligned}
$$

When restricted to $T$, these make a complete system of functions for $H^{1}(T)$. Furthermore, these are orthogonal in $L_{2}(\Omega)$, and also orthogonal with respect to the bilinear form $(\nabla \cdot \nabla \cdot)_{\Omega}$ (and in $\left.H^{1}(\Omega)\right)$. 
$3^{\circ}$. From (b), the condition for a symmetric $\tilde{v} \in H^{1}(\Omega)$ to belong to $\tilde{V}_{2}^{s}$ is expressed by

$$
\begin{array}{r}
2 a_{00}+\sum_{m=1}^{\infty}(-1)^{m} a_{m 0}=0 \text { for } \tilde{v}=\sum_{m \geq n \geq 0}^{\infty} a_{m n} \psi_{m n} \\
\text { with } \sum_{m \geq n \geq 0}^{\infty}\left(1+m^{2}+n^{2}\right) a_{m n}^{2}<+\infty
\end{array}
$$

where $a_{m n}$ 's are real coefficients and the series $\sum_{m=1}^{\infty}(-1)^{m} a_{m 0}$ is shown to be absolutely convergent. Eliminating $a_{00}$ by this condition, we can express $\forall \tilde{v} \in \tilde{V}_{2}^{s}$ by

$$
\tilde{v}=\sum_{m=1}^{\infty} a_{m 0}\left[\psi_{m 0}-(-1)^{m}\right]+\sum_{m \geq n \geq 1}^{\infty} a_{m n} \psi_{m n}
$$

Clearly, $\psi_{m n}$ 's for $m \geq n \geq 1$ are eigenfunctions of (a) with completely homogeneous Neumann's boundary condition, and the minimum of the associated eigenvalues is $2 \pi^{2}$.

$4^{\circ}$. Taking notice of (c), $\tilde{V}_{2}^{s}$ is expressed by the direct sum

$$
\tilde{V}_{2}^{s}=W_{1} \oplus W_{2},\left\{\begin{array}{cc}
W_{1}=\text { closure of linear combinations of } & \psi_{m 0}-(-1)^{m} \\
& (m=1,2,3, \ldots) \\
W_{2}=\text { closure of linear combinations of } \psi_{m n} & (m \geq n \geq 1) .
\end{array}\right.
$$

Here, $W_{1}$ and $W_{2}$ are orthogonal to each other in both $L_{2}(\Omega)$ and $H^{1}(\Omega)$, and moreover, from the observation in $3^{\circ}$, all the eigenfunctions in $W_{2}$ are known. Consequently, our aim will be attained if we obtain the minimum of eigenvalues associated with eigenfunctions in $W_{1}$ : If it is smaller than $2 \pi^{2}$, the obtained one is nothing but $\lambda_{0}$.

$5^{\circ}$. Let us now solve the eigenvalue problem (a) in $W_{1}$ by expressing $\tilde{u} \in$ $W_{1} \backslash\{0\}$ as

$$
\tilde{u}=\sum_{m=1}^{\infty} a_{m} \varphi_{m} \text { with } \sum_{m=1}^{\infty} m^{2} a_{m}^{2}<+\infty ; \quad \varphi_{m}=\psi_{m 0}-(-1)^{m} \quad(m \in \mathbf{N}) .
$$

More specifically, $\varphi_{m}\left(x_{1}, x_{2}\right)=\cos m \pi x_{1}+(-1)^{m}\left(\cos m \pi x_{2}-1\right)$. Substituting (d) into (a) and equating $\tilde{v}$ to each of $\varphi_{m}$ 's, we have the equations for coefficients $a_{m}$ 's:

$$
\left(m^{2} \pi^{2}-\lambda\right) a_{m}=\lambda(-1)^{m} \sum_{n=1}^{\infty}(-1)^{n} a_{n} \quad(m \in \mathbf{N}) .
$$


The series above is absolutely convergent from (d). In addition,

$$
\sum_{n=1}^{\infty}(-1)^{n} a_{n} \neq 0, \lambda \neq m^{2} \pi^{2} \text { and } a_{m} \neq 0 \quad(\forall m \in \mathbf{N})
$$

so that $a_{m}=\lambda(-1)^{m} \sum_{n=1}^{\infty}(-1)^{n} a_{n} /\left(m^{2} \pi^{2}-\lambda\right)$. Multiplying $(-1)^{m}$ to both sides of this equation and summing up for $m \in \mathbf{N}$, we find that

$$
\sum_{m=1}^{\infty}(-1)^{m} a_{m}=\left[\sum_{n=1}^{\infty}(-1)^{n} a_{n}\right] \times \sum_{m=1}^{\infty} \lambda /\left(m^{2} \pi^{2}-\lambda\right),
$$

that is,

$$
1=\sum_{m=1}^{\infty} \frac{\lambda}{m^{2} \pi^{2}-\lambda}, \quad \text { or } \quad \sum_{m=0}^{\infty} \frac{1}{m^{2}(\pi / \sqrt{\lambda})^{2}-1}=0,
$$

where we have used the fact that $\lambda>0$, and the series is absolutely convergent at least for $0<\lambda<\pi^{2}$. We have thus shown that the considered eigenvalue $\lambda$ must satisfy (f). Conversely, for each positive solution $\lambda$ of (f), we can prove in view of (e) the existence of $\tilde{u} \in W_{1} \backslash\{0\}$ that satisfies (a), although we omit the proof.

$6^{\circ}$. Notice here the formula for non-integer $a \in \mathbf{R}$ :

$$
\sum_{m=1}^{\infty} \frac{1}{(m / a)^{2}-1}=\frac{1}{2}-\frac{\pi a}{2 \tan \pi a}
$$

which is for example derived from the Fourier cosine expansion of $\cos a t$ on the interval $[-\pi, \pi]$ for $t$. Comparing the above with (f) and taking $a$ as $\sqrt{\lambda} / \pi$, we have (9). Clearly, the minimum positive solution of (9) lies in the interval $\left(\pi^{2} / 4, \pi^{2}\right)$, and is the unique solution there. It is surely smaller than $2 \pi^{2}$, and is exactly $\lambda_{0}$.

$7^{\circ}$. To obtain $\sqrt{\lambda}_{0} \in(\pi / 2, \pi)$ numerically with verification, we can use various methods. For example, we can directly use the series in (f). Here we just give another method based on modification of the equation $t+\tan t=0$ for $t>0$ : Let us find the minimum positive zero of

$$
f(t):=\frac{\cos t}{2}+\frac{\sin t}{2 t}=\sum_{m=0}^{\infty} \frac{(-1)^{m}(m+1) t^{2 m}}{(2 m+1) !} \quad(t>0) .
$$

The series appearing above is an alternating one, and the absolute value of each term for fixed $t$ converges to 0 as $m \rightarrow \infty$, monotonically for sufficiently large $m$. Moreover, $f(t)$ is monotonically decreasing for $0<t<\pi$. Thus, as is well known in elementary calculus, we can compute upper and lower bounds for the minimum zero $t_{0}$ by utilizing appropriate partial sums: $f_{n}(t):=$ partial sum up to the term of $m=n$. It should be noted here that, at least in principle, all the computations can be performed in the finite-digit binary arithmetic without computer errors, 
provided that $t$ is a rational number. For example, by taking $n=4,5$, we can bound $t_{0}=\sqrt{\lambda}_{0}$ as $2.0287<t_{0}<2.0291$, since $f(2.0291)<f_{4}(2.0291)<0$ (even $n)$ and $f(2.0287)>f_{5}(2.0287)>0($ odd $n)$.

REMARK. Eq. (9) can be also derived as follows. The function $\varphi_{m}$ in (d) can be also expressed by $\varphi_{m}\left(x_{1}, x_{2}\right)=\cos m \pi x_{1}+\cos m \pi\left(1-x_{2}\right)-(-1)^{m}$, so that $\tilde{u} \in W_{1}$ in (d) must be of the form, for an unknown single-variable function $g=g(t)$

$$
\tilde{u}\left(x_{1}, x_{2}\right)=g\left(x_{1}\right)+g\left(1-x_{2}\right) .
$$

Substituting the above into (a), we have

$$
-g^{\prime \prime}(t)=\lambda g(t) \text { for } 0<t<1, \quad g^{\prime}(0)=0, \quad g(1)+\int_{0}^{1} g(t) d t=0 .
$$

Notice in this derivation that $\tilde{v}$ in (a) can be taken from whole $\tilde{V}_{2}$, since $W_{1}$ is orthogonal to $W_{2}$ and $V_{2}^{a}$ both in $L_{2}(\Omega)$ and $H^{1}(\Omega)$. Solving this eigenvalue problem, we obtain (9). Moreover, an eigenfunction associated to $\lambda_{0}$ is $\tilde{u}\left(x_{1}, x_{2}\right)=$ $\cos \sqrt{\lambda}_{0} x_{1}+\cos \sqrt{\lambda}_{0}\left(1-x_{2}\right)$. Eq. (9) is popular in vibration analysis of a string with one end fixed and the other supported elastically, where the governing differential equation is the same as in (11).

\section{Concludig Remarks}

We have succeeded in determining the Babuska-Aziz constant from a very simple equation. We can effectively utilize this constant to give upper bounds of the $P_{1}$-interpolation error constants for triangles of more general shape. That is, we can derive some explicit relations for the dependence of such constants on the geometry (such as the maximum interior angle and the minimum edge length) of triangles by simple coordinate transformations. It is to be noted that they are consistent with the so-called maximum angle condition in [2]. The detailed results will be reported separately in due course.

Acknowledgements. The authors would like to express their deepest appreciation to Prof. M.T. Nakao of Graduate School of Mathematical Sciences, Kyushu University and Prof. N. Yamamoto of Department of Computer Science, The University of Electro-Communication for acquainting them with the importance of the present problem and a number of references. Some of the exact computations in this paper were performed with the aid of Mathematica ${ }^{\circledR} 5$. This work was partially supported by Japan Society for the Promotion of Science Grant-in-Aid for Scientific Research (C) (2) 16540096, and also by Yoshida Scholarship Foundation for the second author.

\section{References}

[1] P. Arbenz, Computable finite element error bounds for Poisson's equation. IMA J. Numer. Anal., 2 (1982), 475-479. 
[ 2 ] I. Babuska and A.K. Aziz, On the angle condition in the finite element method. SIAM J. Numer. Anal., 13 (1976), 214-226.

[ 3 ] R.E. Bahnhill, J.H. Brown and A.R. Mitchell, A comparison of finite element error bounds for Poisson's equation. IMA J. Numer. Anal., 1 (1981), 95-103.

[ 4 ] S.C. Brenner and L.R. Scott, The Mathematical Theory of Finite Element Methods (2nd edn.). Springer, 2002.

[ 5 ] P.-G. Ciarlet, The Finite Element Method for Elliptic Problems. SIAM, 2002.

[6] P. Knabner and L. Angermann, Numerical Methods for Elliptic and Parabolic Partial Differential Equations. Springer, 2003.

[ 7 ] R. Lehmann, Computable error bounds in finite-element method. IMA J. Numer. Anal., 6 (1986), 265-271.

[ 8 ] M.T. Nakao and N. Yamamoto, A guaranteed bound of the optimal constant in the error estimates for linear triangular element. Computing [Supplementum], 15 (2001), 163-173.

[ 9 ] M.T. Nakao and N. Yamamoto, A guaranteed bound of the optimal constant in the error estimates for linear triangular elements, Part II: Details. Perspectives on Enclosure Methods (eds. U. Kulisch et al.), the Proceedings Volume for Invited Lectures of SCAN2000, SpringerVerlag, Vienna, 2001, 265-276.

[10] F. Natterer, Berechenbare Fehlerschranken für die Methode der finite Elemente. International Series of Numerical Mathematics, 28, Birkhäuser, 1975, 109-121.

[11] G.L. Siganevich, On the optimal estimation of error of the linear interpolation on a triangle of functions from $W_{2}^{2}(T)$ (in Russian). Doklady Akademii Nauk SSSR, 300, No.4 (1988), $811-814$. 